\title{
CHARA/MIRC OBSERVATIONS OF TWO M SUPERGIANTS IN PERSEUS OB1: TEMPERATURE, BAYESIAN MODELING, AND COMPRESSED SENSING IMAGING
}

\author{
F. Baron ${ }^{1,2}$, J. D. Monnier ${ }^{1}$, L. L. Kiss ${ }^{3,4}$, H. R. NeIlson ${ }^{5}$, M. Zhao ${ }^{6}$, M. Anderson ${ }^{1}$, A. Aarnio $^{1}$, E. Pedretti $^{7}$, \\ N. Thureau ${ }^{7}$, T. A. ten Brummelaar ${ }^{8}$, S. T. Ridgway $^{9}$, H. A. McAlister ${ }^{2,8}$, J. Sturmann $^{8}$, \\ L. StURMANN ${ }^{8}$, AND N. TURNER ${ }^{8}$ \\ ${ }^{1}$ Department of Astronomy, University of Michigan, 918 Dennison Building, Ann Arbor, MI 48109-1090, USA; baron@ phy-astr.gsu.edu \\ ${ }^{2}$ CHARA and Department of Physics and Astronomy, Georgia State University, P. O. Box 4106, Atlanta, GA 30302-4106, USA \\ ${ }^{3}$ Sydney Institute for Astrophysics, School of Physics, University of Sydney, NSW 2006, Australia \\ ${ }^{4}$ Konkoly Observatory, Hungarian Academy of Sciences, H-1525 Budapest, Hungary \\ ${ }^{5}$ Department of Physics and Astronomy, East Tennessee State University, Box 70652, Johnson City, TN 37614, USA \\ ${ }^{6}$ Department of Astronomy and Astrophysics, Penn State University, University Park, PA 16802, USA \\ ${ }^{7}$ Department of Physics and Astronomy, University of St. Andrews, UK \\ ${ }^{8}$ The CHARA Array, Georgia State University, P.O. Box 3965, Atlanta, GA 30302-3965, USA \\ ${ }^{9}$ National Optical Astronomy Observatory, Tucson, AZ 85726-6732, USA \\ Received 2013 June 10; accepted 2014 January 27; published 2014 March 25
}

\begin{abstract}
Two red supergiants (RSGs) of the Per OB1 association, RS Per and T Per, have been observed in the $H$ band using the Michigan Infra-Red Combiner (MIRC) instrument at the CHARA array. The data show clear evidence of a departure from circular symmetry. We present here new techniques specially developed to analyze such cases, based on state-of-the-art statistical frameworks. The stellar surfaces are first modeled as limb-darkened disks based on SATLAS models that fit both MIRC interferometric data and publicly available spectrophotometric data. Bayesian model selection is then used to determine the most probable number of spots. The effective surface temperatures are also determined and give further support to the recently derived hotter temperature scales of RSGs. The stellar surfaces are reconstructed by our model-independent imaging code SQUEEZE, making use of its novel regularizer based on Compressed Sensing theory. We find excellent agreement between the model-selection results and the reconstructions. Our results provide evidence for the presence of near-infrared spots representing about 3\%-5\% of the stellar flux.
\end{abstract}

Key words: stars: fundamental parameters - starspots - supergiants - techniques: image processing - techniques: interferometric

Online-only material: color figures

\section{INTRODUCTION}

Red supergiants (RSGs) represent an important but still poorly characterized evolutionary phase of massive stars. As He-burning evolved stars, their surfaces present very cool effective temperatures between 3400 and $4100 \mathrm{~K}$ (spectral type of late-K to M) and average luminosities 20,000-300,000 $L_{\odot}$ (Levesque et al. 2005; Levesque et al. 2006). RSGs are among the largest stars, with radii up to $1500 R_{\odot}$ and masses in the 10-25 $M_{\odot}$ range (Levesque et al. 2005; Massey et al. 2008; Levesque 2010). The resulting low gravity has the consequence that material from the outer envelope can easily escape to the interstellar medium, giving rise to circumstellar envelopes (Danchi et al. 1994) and very significant mass-loss rates ranging between $10^{-8}$ to $10^{-4} M_{\odot} \mathrm{yr}^{-1}$ (Massey et al. 2005; Verhoelst et al. 2009; Sargent et al. 2011). The actual mechanism of the mass loss is still unknown but is likely to involve the combined effects of turbulent pressure from large convection cells and radiation pressure on molecular lines (Josselin \& Plez 2007), as well as stellar magnetism (Grunhut et al. 2010; Aurière et al. 2010) and Alfvén winds (Cuntz 1997; Airapetian et al. 2010). Through these processes, RSGs are key agents of nucleosynthesis and chemical enrichment of the Galaxy.

In the last decade, photometric and spectroscopic observations have allowed several breakthroughs in our understanding of their dynamical convective patterns. RSGs have long been known for their semi-regular short-term optical variations with periods of the order of hundreds of days (Kiss et al. 2006). Historically, this variability was usually attributed to radial pulsation, and while this path is still being investigated (Yang \& Jiang 2012), the full explanation is now thought to involve variation of a few large granules on the surface of the RSGs. Such huge convection cells are suggested by theory and simulations (Schwarzschild 1975; Stothers 2010), and supported by recent spectroscopic observations that detected large amounts of material moving through the photosphere of RSGs (Josselin \& Plez 2007; Gray 2008; Ohnaka et al. 2009, 2011, 2013).

Modeling these convection effects requires a precise determination of the temperature of the RSGs. The last decade of measurements of effective temperatures of RSGs seemed to indicate much cooler temperatures than predicted by stellar evolutionary theory, until Levesque et al. (2005) used MARCS stellar atmosphere models with state-of-the art (at the time) treatment of molecular opacities (Gustafsson et al. 1975; Plez 2003) to fit moderate-resolution optical spectrophotometry of Galactic RSGs. They derived a warmer effective temperature scale for RSGs of Galactic metallicity than previous studies, in rough agreement with the Geneva evolutionary tracks (Meynet \& Maeder 2003; Levesque et al. 2006). Long-baseline interferometry brings unique insights to the study of RSGs. Because optically resolving RSGs by interferometry gives direct access to their angular diameters, it is complementary to spectrophotometry. In a recently published survey of 74 RSGs with the Palomar Testbed Interferometer (PTI), van Belle et al. (2009) 
thus derived a slightly warmer temperature scale than that of Levesque et al. (2005).

Convection processes are involved in the creation of hotspots, and determining how the formation and evolution of hotspots is correlated to the fundamental stellar parameters is a difficult task. The presence of spots and large convection cells affects the estimation of these fundamental parameters, as it leads to shortterm photometric variability (Chiavassa et al. 2011) in addition to long-term effects (Kiss et al. 2006), while also producing photocenter shifts that may bias diameter determination (Chiavassa et al. 2009).

High angular resolutions techniques are becoming essential tools to understand these hotspots. Using aperture masking at the William Herschel Telescope, Buscher et al. (1990) and Tuthill et al. (1997) found bright asymmetries at visible wavelengths on the surface of M-supergiants ( $\alpha$ Ori $\alpha$ Sco, $\alpha$ Her), with timescale variations of order a few months possibly explained by the presence of hotspots. Because of its large angular size, $\alpha$ Ori then became the best-studied individual RSG in terms of multiwavelength surface imaging. Using the COAST interferometer, Young et al. (2000) found a strong variation in the apparent asymmetry as a function of wavelength, with the detection of hotspots in the visible, but only featureless disk in $J$ band. This has led to the suggestion that the bright spots are unobscured regions of elevated temperature, seen through a geometrically extended and line-blanketed atmosphere, in which the features are seen along lines of sight for which the atmospheric opacity has been reduced as the result of activity (e.g., convection) at the stellar surface. In $H$ or $J$ band, the continuum opacity is close to minimum in these cool atmospheres (Woodruff et al. 2009), one would expect to see the photosphere, with negligible or no evidence of hotspots at this band.

However, interferometric observations of asymptotic giant branch (AGB) stars (somewhat less massive and less luminous than RSGs) have revealed that a significant fraction of these present strong closure phase signals (Ragland et al. 2006). These signals may be explained by unresolved bright spots, though circumstellar emission could not be ruled out. While the envelopes and dust shells of several RSGs have been successfully imaged and shown to be very often asymmetric (Monnier et al. 2004b; Kervella et al. 2011), resolving actual surface features has proved more difficult. It is only recently that Haubois et al. (2009) reported the unambiguous detection of two hotspots on $\alpha$ Ori by the IOTA interferometer in $H$ band, while Chiavassa et al. (2010b) found a similar number of spots in the same band on VX Sgr using with VLTI/AMBER. These spots are thought to be the imprint of giant convection cells based on three-dimensional (3D) stellar convection models (Freytag et al. 2002; Chiavassa et al. 2009; Stothers 2010; Chiavassa et al. 2010a).

We present in Section 2 of this paper our observations of two RSGs from the Per OB1 association, T Per and RS Per, using the world-leading resolution of the Center for High Angular Resolution Astronomy (CHARA) array to resolve their surface in $H$ band. Then in Section 3, we attempt to model the stellar surface asymmetries as spots, and we devise a procedure to determine the probabilities of these models based on state-ofthe-art Bayesian techniques. Using our best estimates of the stellar diameters, we then derive the linear sizes, bolometric fluxes, and temperatures of both stars. Finally in Section 4, we present model-independent images of both RSGs obtained with the latest version of the software SQUEEZE and a new regularizer developed for spotted star reconstruction.

\section{OBSERVATIONS}

\subsection{RS Per and T Per}

In the following, we will present our observations of two Red Supergiants from the Per OB1 association, RS Per (HD 14488) and T Per (HD 14142). RS Per is a firmly established member of the $\chi$ Per/NGC 884 cluster, while T Per lies about $2^{\circ}$ north above the double cluster (Lee \& Lim 2008). Both are M supergiants, with RS Per classed as M4I, and T Per as M2I. Based on averaged previous results (Gonzalez \& Wallerstein 2000; Slesnick et al. 2002; Levesque et al. 2005), T Per has a temperature typical of most M supergiants in the Per OB1 association (average temperature in the literature $T \simeq 3850 \mathrm{~K}$ ), while RS Per is thought to be slightly cooler ( $T \simeq 3500 \mathrm{~K}$ ). It is interesting to note that they both display comparable long photometric periods, $2500 \pm$ 460 days for T Per, $4200 \pm 1500$ days for RS Per (Kiss et al. 2006; Percy \& Sato 2009), thought to be related to a global pulsation mode. RS Per also displays a shorter period of 245 days.

\subsection{CHARA/MIRC Observations}

Our observations were carried out on five nights in 2007 July-August at the Georgia State University CHARA interferometer array using the Michigan Infra-Red Combiner (MIRC). The CHARA array, located on Mount Wilson, consists of six $1 \mathrm{~m}$ telescopes. Thanks to its 15 baselines ranging from $34 \mathrm{~m}$ to $331 \mathrm{~m}$, it achieves the highest angular resolution of optical interferometers, up to $\sim 0.5$ mas in $H$ band (ten Brummelaar et al. 2005).

The MIRC instrument was used to combine the $H$-band light $(1.5-1.8 \mu \mathrm{m})$ of four CHARA telescopes at low spectral resolution $(R=30)$. This provided six visibilities, four closure phases, and four triple amplitudes simultaneously in each of the eight $30 \mathrm{~nm}$ wide spectral channels (Monnier et al. 2004a; Monnier et al. 2006). Using the same W1-W2-S2-E2 configuration of CHARA that was used for surface imaging of Altair (Monnier et al. 2007), we achieved adequate $(u, v)$ coverage of each target for imaging, as shown in Figure 1. The longest baseline in this configuration is $251.3 \mathrm{~m}$, corresponding to a resolution of 1.3 mas at $1.6 \mu \mathrm{m}$. We secured four data blocks for RS Per and three for T Per, each data block corresponding to a continuous observation of a target during about 20 minutes.

The data were reduced by the latest version of the MIRC pipeline written by John Monnier (as of 2012 November) and previously described in Monnier et al. (2007). The pipeline computes the squared visibilities using Fourier transforms, then averages them. The bispectrum is formed using the phases and amplitudes of three baselines that form a closed triangle. For each data block, we use the best method available for amplitude calibration: for T Per the fluxes were estimated by the chopper method and for RS Per both the chopper and DAQ method (Monnier et al. 2008). Our targets were observed along reference calibrators to correct for the usual transfer function variations that occur during the night due to atmospheric and optical changes in the beam path (Perrin 2003). Our observations were typical of July-August weather, with transfer functions remaining very stable (less than 0.2 drop in visibility during the nights).

The calibrators were modeled as uniform disks as indicated in Table 1. Note that 37 And has recently been resolved by MIRC as a binary; however, the flux ratio of the components is greater than 1:100 and thus this does not significantly impact our calibration. 

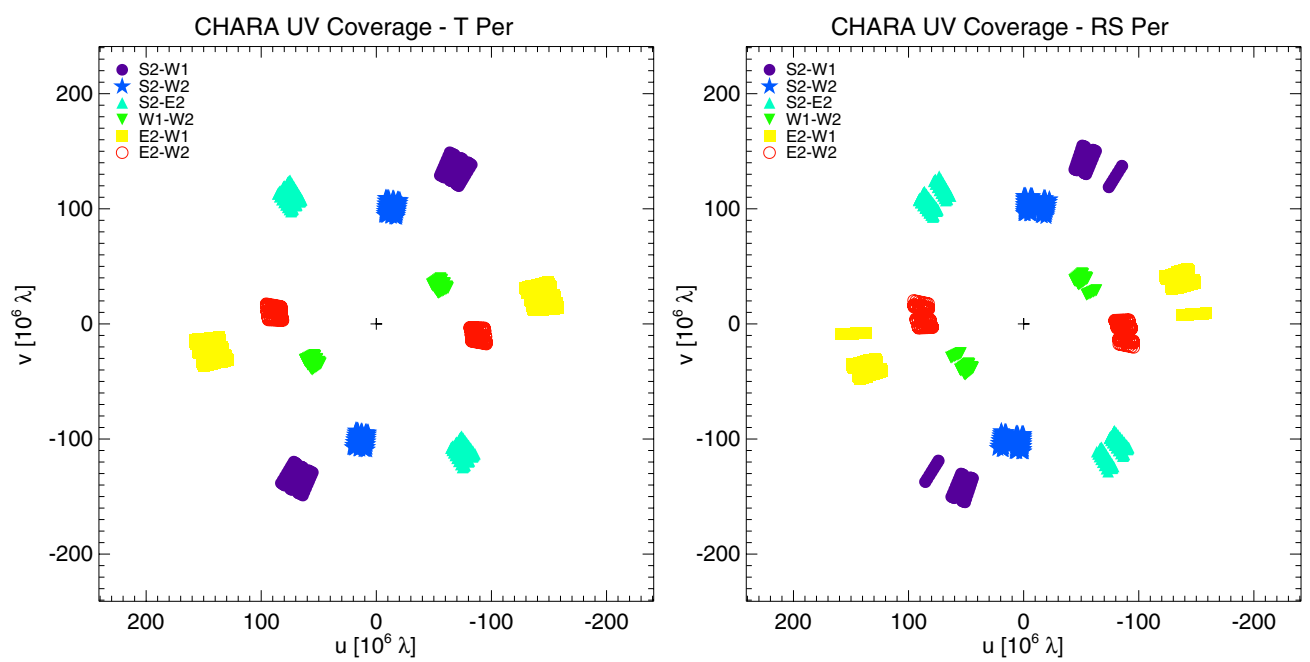

Figure 1. Combined $(u, v)$ coverage of our observations of T Per (left) and RS Per (right). The telescope configuration used was S2-E2-W1-W2. The radial dispersion is due to the use of MIRC low spectral mode with eight channels.

(A color version of this figure is available in the online journal.)

Table 1

Reduction $\log$ for T Per and RS Per

\begin{tabular}{lcccc}
\hline \hline $\begin{array}{l}\text { Date } \\
\text { (UT) }\end{array}$ & Target & $N_{\text {block }}$ & Calibrators & Flux Calibration \\
\hline 2007 Jul 28 & T Per & 2 & HD 9022 & Chopper \\
2007 Jul 29 & T Per & 1 & $v$ And & Fiber \\
2007 Jul 30 & RS Per & 1 & $v$ And & Chopper \\
2007 Jul 31 & RS Per & 1 & 37 And & DAQ \\
2007 Aug 2 & RS Per & 2 & $\sigma$ Cyg, $v$ And & DAQ \\
\hline
\end{tabular}

Notes. $N_{\text {block }}$ refers to the number of data blocks. All calibrated OI-FITS data are available upon request. Calibrator diameters in milliarcseconds: HD $9022=$ $1.05 \pm 0.02$ (Mérand et al. 2005). $v$ And $=1.097 \pm 0.009$ (Zhao et al. 2011). 37 And $=0.676 \pm 0.034$ (Kervella \& Fouqué 2008). $\sigma$ Cyg $=0.542 \pm 0.021$ (Barnes et al. 1978).

As the brightness distributions of both targets is not expected to vary significantly during our observing run (see Table 1 for the exact observing dates), all nights were combined into a single data file for each target, resulting in data sets that total 419 power spectra and 264 bispectra for T Per and 523 power spectra and 326 bispectra for RS Per. Systematic errors are taken into account by applying additive and multiplicative errors on the data. All the following nominal values were determined based on the expertise of the MIRC group with MIRC 2007 data (J. Monnier 2013, private communication), and based on the in-depth study of $v$ And data acquired during the same nights (Zhao et al. 2011). Additive errors, that correct for biases at low fringe contrast were set to $2 \times 10^{-4}$ for squared visibilities and $10^{-5}$ for triple amplitudes. Multiplicative errors, that correct for the uncertainties in the transfer function, were $20 \%$ on squared visibilities and $30 \%$ on triple amplitudes. Based on the analysis of the closure phase statistics made by Zhao et al. (2011), an error floor of $1^{\circ}$ is chosen on closure phases. To account for the unreliability of closure estimation at low flux, each closure error is further increased by $30^{\circ} / S_{\mathrm{T} 3 \mathrm{amp}}^{2}$, where $S_{\mathrm{T} 3 \mathrm{amp}}$ is the signal-tonoise of the corresponding triple amplitude. To account for the difficulty of estimating rapidly varying closures, an error equal to $10 \%$ of the closure gradient in the spectral domain is normally added to MIRC closures. However for these data, the closures barely vary with wavelength, and this effect is negligible.
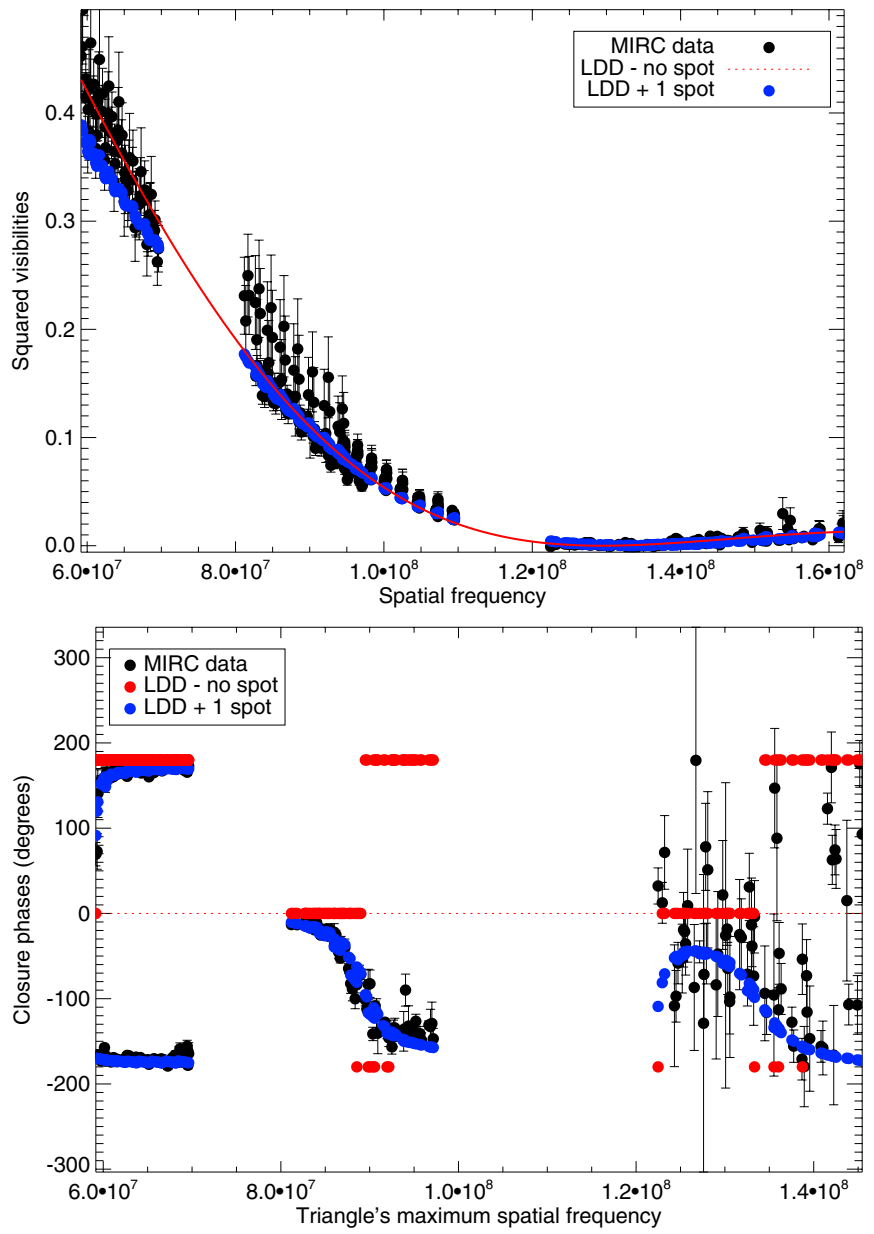

Figure 2. Power spectrum and closure phase fits for T Per. (A color version of this figure is available in the online journal.)

\section{MODEL-FITTING}

All the available square visibilities and closure phases are plotted on Figures 2 and 3 as a function of their spatial frequency. The power spectrum curves are typical of limb-darkened stellar disks, while the closure phases clearly depart from zero, 

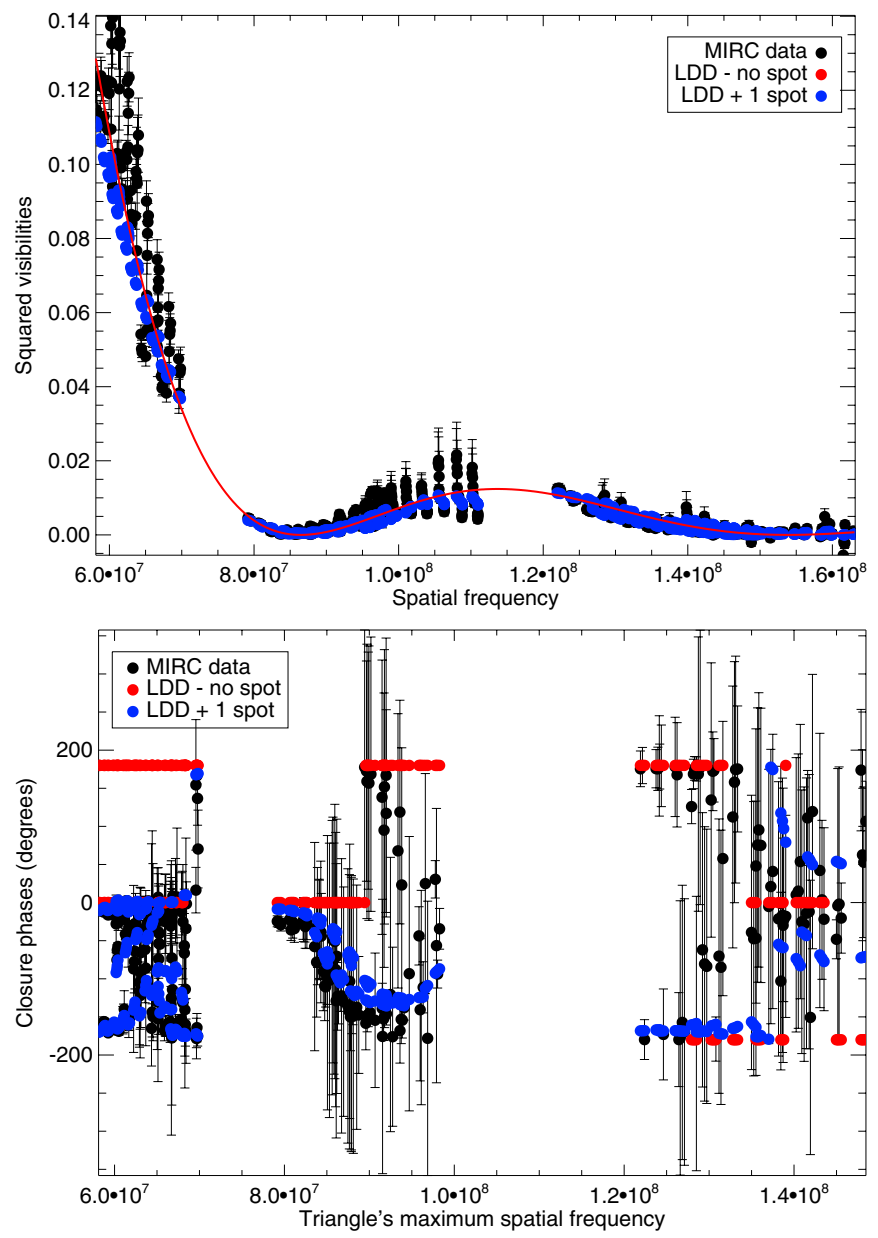

Figure 3. Power spectrum and closure phase fits for RS Per.

(A color version of this figure is available in the online journal.)

indicating the presence of strong resolved asymmetries on the stellar surfaces. Based on the previous interferometric results on $\mathrm{M}$ supergiants in the literature, we expect these to be due to the presence of spots.

\subsection{Spotless Models: Limb-darkening}

Before attempting a spot search, we first sought to roughly characterize the size and brightness distribution of the stellar disks. Our model-fitting code FITNESS (F. Baron et al. 2014, in preparation) was used to fit several limb-darkening models (square root, quadratic, power law) to the power spectra and triple amplitudes. FITNESS allows the use of various minimization engines, and here it was set to employ a straightforward combination of grid search to identify the global $\chi^{2}$ minima and of Levenberg-Marquardt on each grid point to refine the parameters. The best fits were obtained for the linear law and the Hestroffer power law (Hestroffer 1997), but they both show the existence of a strong covariance between the limb-darkened angular diameter and the limb-darkening coefficient. The issue is illustrated on Figure 4 (top), where the reduced $\chi^{2}$ surface is plotted as a function of both parameters. For both targets, the problem is mainly due to the lack of high signal-to-noise data on the first visibility lobes. To increase the precision of the fit on the angular diameter, the limb-darkening coefficients have to be constrained.

Haubois et al. (2009) reported successfully fitting the visibility curve of $\alpha$ Ori (M2 type, $T_{\text {eff }} \simeq 3600 \mathrm{~K}$ ) with a linear

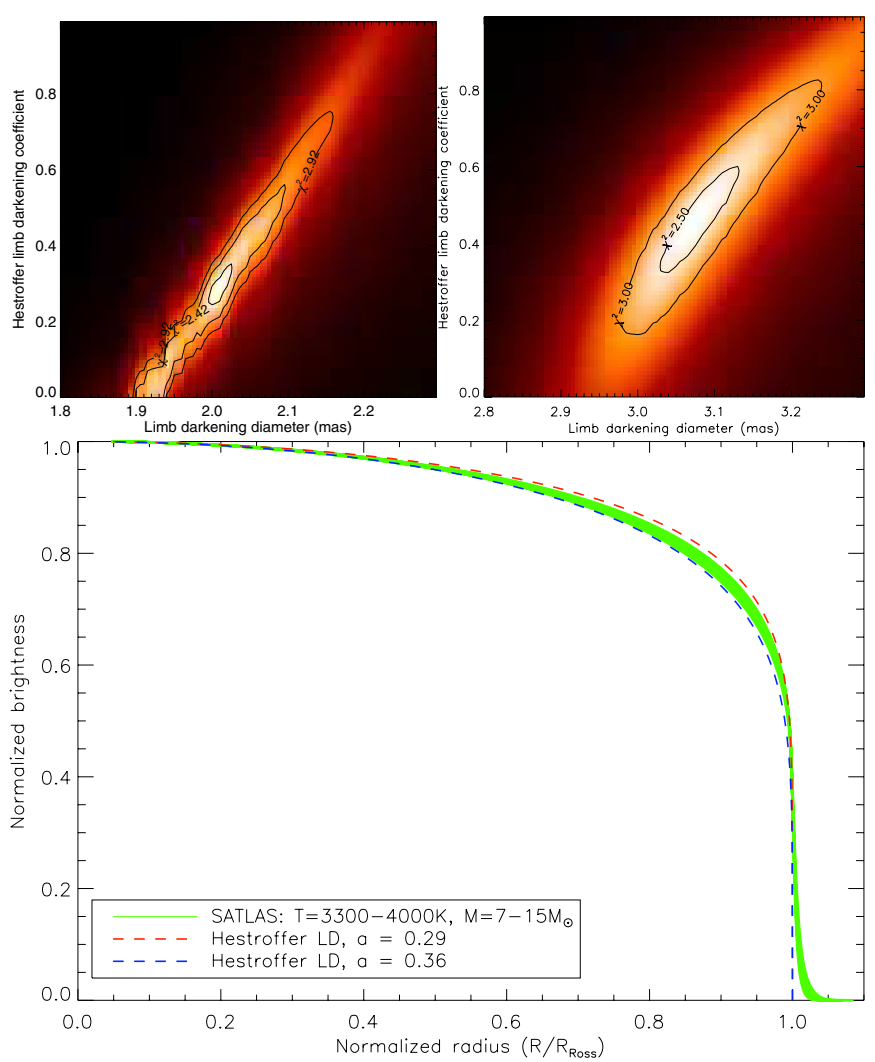

Figure 4. Constraints on the limb-darkening. Top panel: $\chi^{2}$ surface for the linearly limb-darkened disks of T Per (left) and RS Per (right) based on interferometric data. Bottom panel: SATLAS limb-darkening models for a range of temperature, gravity, and mass values compatible with previous observations of RS Per and T Per.

(A color version of this figure is available in the online journal.)

coefficient $\alpha=0.43 \pm 0.03$ (roughly corresponding to a Hestroffer law with coefficient $0.3-0.4$ ). Beyond this empirical result, it seems non-obvious whether conventional plane-parallel one-dimensional radiative codes such as ATLAS (Kurucz 1992; Castelli \& Kurucz 2003) or MARCS (Gustafsson et al. 1975) may reliably predict the intensity profiles of RSGs. RSGs are notoriously difficult to model: their atmospheres are very extended, which invalidates the assumption of plane-parallel geometry, and their very cool temperatures require an advanced treatment of molecular opacities. However, more recent codes that assume spherical geometry such as MARCS-spherical (Gustafsson et al. 2008), SATLAS (Lester \& Neilson 2008), and PHOENIX (Hauschildt \& Baron 1999) have demonstrated successful results on comparably cool $\mathrm{M}$ giants or supergiants (Wittkowski et al. 2004, 2006, 2012).

In order to constrain the limb-darkening in $H$ band for both RSGs, we used the latest SATLAS code (available at http://www.astro.utoronto.ca/ lester/programs.html), which includes improved ODF treatment and fixed $\mathrm{H}_{2} \mathrm{O}$ lines. Model parameters were based around the values found in Gonzalez \& Wallerstein (2000) and Slesnick et al. (2002): temperatures ranging from $3100 \mathrm{~K}$ to $4000 \mathrm{~K}$ ( steps of $100 \mathrm{~K}$ ), $\log g=-0.5$ to 0.5 and a fixed metallicity $[\mathrm{Fe} / \mathrm{Z}]=-0.5$ (Gonzalez \& Wallerstein 2000). We also used MARCS models as cross-checks but found negligible difference with SATLAS ones. Figure 4 (bottom) presents the results of these simulations as a band of possible brightness distributions. The intensity profiles are weakly dependent on the temperature and are mostly determined by the mass and surface gravity. They are characteristics of spherical 
Table 2

Model-fitting Results for T Per (Angles given East of North)

\begin{tabular}{lcccc}
\hline \hline Fit Results & No Spot & Dark Spot & Bright Spot & Two Spots \\
\hline$\chi_{r}^{2}$ & 9.4 & 2.24 & 2.64 & 1.95 \\
$\log Z( \pm 0.02)$ & -0.35 & 0.87 & 1.23 & 0.20 \\
$\theta_{\star}($ mas $)$ & 2.01 & 2.02 & 2.02 & 2.02 \\
$f_{\text {spot }}$ & $\ldots$ & $4 \%$ & $5 \%$ & $3 \%, 4 \%$ \\
$\theta_{\text {spot }}$ (mas) & $\ldots$ & $0.42 \pm 0.03$ & $0.26 \pm 0.01$ & $0.45 \pm 0.03$ \\
& & & & $0.56 \pm 0.03$ \\
$r_{\text {spot }}$ (mas), $\varphi_{\text {spot }}$ & $\ldots$ & $0.21,-135^{\circ}$ & $0.22,134^{\circ}$ & $0.22,134^{\circ}$ \\
& & & & $0.73,238^{\circ}$ \\
\hline
\end{tabular}

Notes. We report the following parameters for the best model: $\chi_{r}^{2}$ : reduced $\chi^{2}$ of the best fit, $\log Z$ : the logarithm of the marginal likelihood (defined in Section 3.3), $\theta_{\star}:$ stellar radius, $\theta_{\star}:$ spot radius, the fractional flux, and the polar coordinates $(r, \varphi)$ of the spot (s).

codes, showing a sudden drop of intensity at the Rosseland radius, where the Rosseland mean opacity equals unity and where most photons escape the atmosphere. As shown on Figure 4, the brightness distributions can adequately be bounded by two Hestroffer laws with coefficients 0.29 and 0.36 . Most limbdarkening laws with two or more parameters, such as the ones suggested by Chiavassa et al. (2009) or Neilson \& Lester (2013), can also fit the distribution well. But, because second lobes are absent from our data sets, the first order laws are more than adequate to model data.

Injecting this prior into the fit, we found the Hestroffer limbdarkening coefficients to be $\alpha=0.32 \pm 0.2$ for $\mathrm{T}$ Per and $\alpha=0.34 \pm 0.2$ for RS Per. Assuming that both RSGs have similar masses, as the angular diameter of $\mathrm{T}$ Per is smaller, we expect a stronger gravity at its surface, which indeed corresponds to a lower limb-darkening coefficient.

Taking into account all statistical errors due to the visibility measurements and the calibration via data bootstrapping, we also obtained the following limb-darkened diameters: for T Per: $\theta_{\mathrm{LD}}=2.01 \pm 0.03$ mas, with $\chi^{2}=1.92$ at the nominal values. For RS Per, $\theta_{\mathrm{LD}}=3.05 \pm 0.05$ mas and with $\chi^{2}=2.37$. Because non-zero closure phases cannot be fitted by a limbdarkening model, the "full" $\chi^{2}$-including the closure phase data-are larger, $\chi^{2}=7.8$ for RS Per and $\chi^{2}=9.4$ for T Per, unambiguously indicating the presence of significant asymmetries on the stellar surfaces.

\subsection{Models with One or Two Spots}

The most economical assumption to explain the closure phases is the presence of spots on the stellar surface. The formation of complex granulations is expected on the surface (Freytag et al. 2002; Chiavassa et al. 2011), and at the resolution and dynamic contrast provided by interferometry, these appear as compact spots. Here we attempted to search only for the most prominent features, with the assumption that the stellar surface could be described as a limb-darkened disk with a limited number of these spots. Previous studies on $\alpha$ Ori demonstrated that spots may be modeled well by Gaussians or uniform ellipses (Young et al. 2000; Haubois et al. 2009). We also chose to use ellipses, so that each spot is modeled by six parameters: its coordinates on the stellar surface, its size, its flux contribution, its orientation angle and its ellipticity. Our priors on these parameters were flat. During model-fitting, spots were not constrained to be brighter than the stellar surface (hot) and thus dark (cold) spots were not ruled out. Spots lying on the circumference of the stellar disk were also searched for.
Table 3

Model-fitting Results for RS Per (Angles given East of North)

\begin{tabular}{lcc}
\hline \hline Fit Results & No Spot & Single Dark Spot \\
\hline$\chi_{r}^{2}$ & 7.8 & 1.2 \\
$\log Z( \pm 0.05)$ & -0.35 & 0.32 \\
$\theta_{\star}$ (mas) & 3.05 & 3.06 \\
$f_{\text {spot }}$ & $\ldots$ & $4 \%$ \\
$\theta_{\text {spot }}$ (mas) & $\ldots$ & $0.80 \pm 0.05$ \\
$r_{\text {spot }}$ (mas),$\varphi_{\text {spot }}$ & $\ldots$ & $1.43,147^{\circ}$ \\
\hline
\end{tabular}

Fitting spots is a difficult numerical problem due to the properties of the $\chi^{2}$. First, while the bispectrum probability density is generally approximated by a convex normal distribution (Meimon et al. 2005; Thiebaut 2008), this approximation breaks down for small triple amplitudes, e.g., for very resolved targets such as these RSGs. Therefore, we revert to using separate $\chi^{2}$ expressions for closures and triple amplitudes. The likelihood expression for the closure phases may then be chosen based $2 \pi$-wrapped normal distribution (Haniff 1991) or the von Mises distribution, and in general is non-convex. Moreover, because the phase information is only partially retrieved from closure phases, the $\chi^{2}$ is multimodal, i.e., there exist local minima into which minimizers can easily get trapped (Meimon et al. 2008). The $\chi^{2}$-minimization strategy has to take both non-convexity and multimodality into account. Because of the relatively large number of parameters (eight to fourteen: two for the stellar disk description, plus six per spot), our strategy consisted in a grid search on the positions of the spots, with a three-step Levenberg-Marquardt minimization at each given position. During the first step, only the stellar disk parameters (diameter and Hestroffer coefficient) and the spot flux are allowed to vary. During the second step, the shape of the spots (size, aspect ratio, orientation) is optimized, then in the third step all the parameters become free to settle.

Despite allowing for potential ellipticity of the stars based on the finding of van Belle et al. (2009), the fits happened to converge on solutions with negligible ellipticities, i.e., $1.0 \pm$ 0.005 . Figures 2 and 3 show that overall our best fits are adequate but clearly underestimate the visibilities on the short baselines. The most probable explanation is a bias in the photometric calibration of the 2007 MIRC data, which tends to occur only on short baselines. We nonetheless investigated other possibilities of a faint resolved shell around the star, modeled as an additional Gaussian component, but this worsened the fit for both stars. The discrepancy at short baselines could still be due to the presence of circumstellar material obscuring the stellar disks near their edge, or just that our assumption of a linearly limb-darkened disk model is too naive, compared to models with a slower decay toward the stellar border.

Note that model-independent image reconstruction, carried out in Section 4, also shows evidence for strong darkening at the periphery. However, these considerations do not significantly affect our spot analysis, relying on medium and long baseline visibilities. Figures 5 and 6 presents the $\chi^{2}$ surface as a function of the spot position, as well as the corresponding best one-spot and two-spot models for T Per and RS Per. In all cases, we found that the total flux contribution of the spots represents roughly $\sim 4 \%$ of the stellar flux, which is comparable to the values found in Haubois et al. (2009) for Betelgeuse spots. The reduced $\chi^{2}$ and parameters for these models are given in Tables 2 and 3, with error bars derived using the classic bootstrapping 

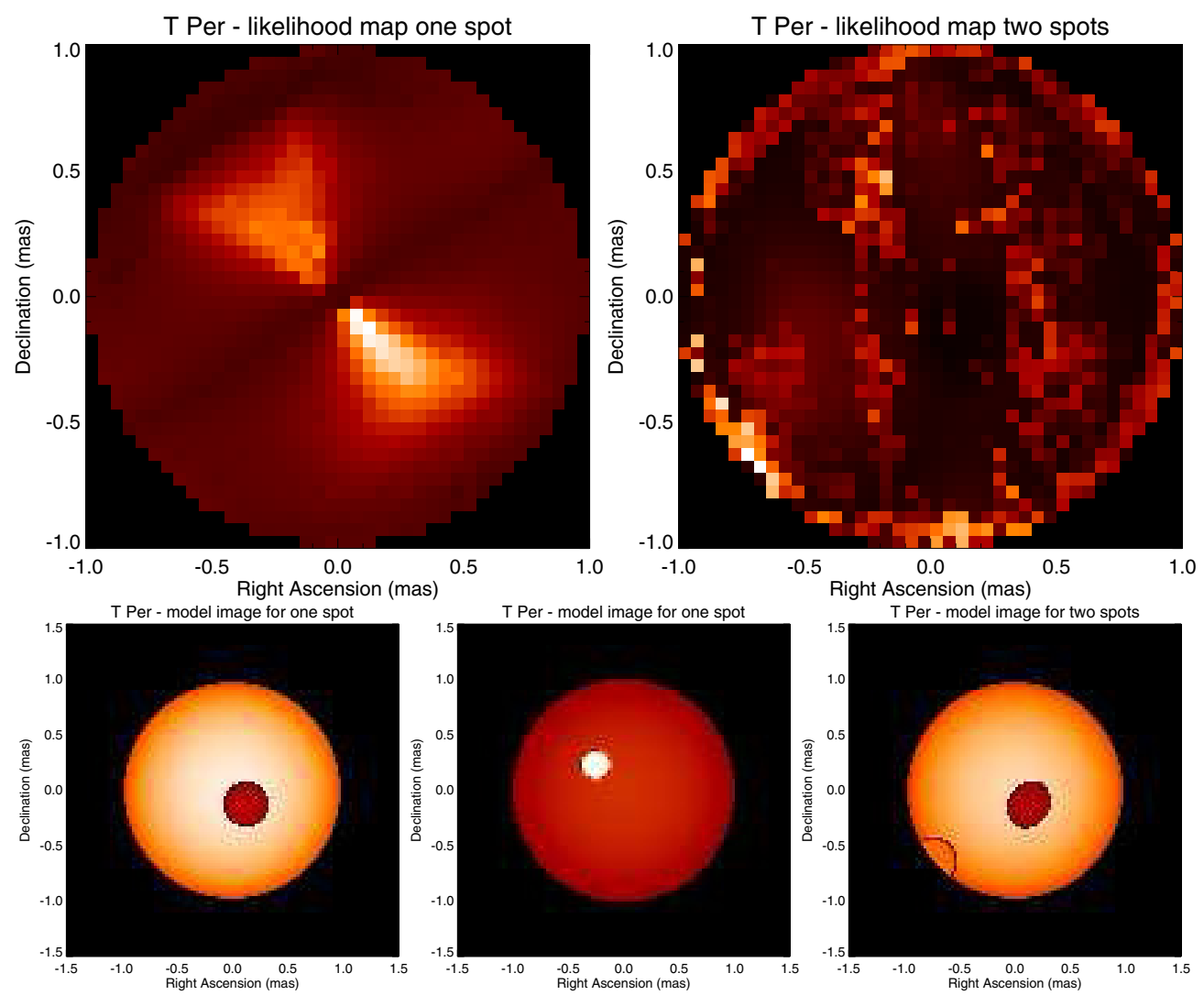

Figure 5. Simple modeling of T Per: likelihood maps for one- and two-spot models (top); corresponding best fitting images for a dark spot, bright spot, and two spots (bottom).

(A color version of this figure is available in the online journal.)
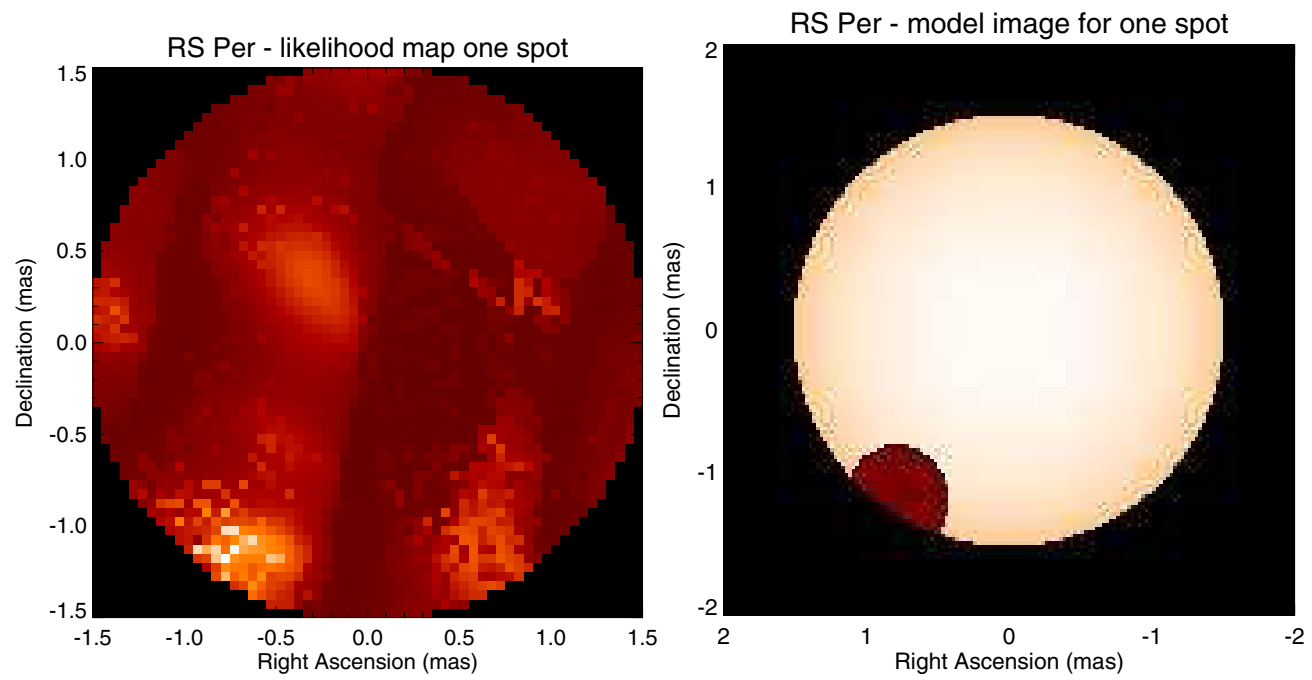

Figure 6. Simple modeling of RS Per: likelihood map of the spot location (left) and best fitting image with a dark spot (right).

(A color version of this figure is available in the online journal.)

technique. For T Per, our results imply the presence of a spot on along the diagonal NW-SE. Based solely on the $\chi^{2}$ metrics, a dark spot in the SE quadrant $\left(\chi^{2}=2.24\right)$, or a bright spot on the NW quadrant seem equally probable $\left(\chi^{2}=2.35\right)$. There is also a slight decrease of $\chi^{2}\left(\chi^{2}=1.9\right)$ when attempting to fit an additional spot to the dark spot model. For RS Per, the results are clearer, with evidence of a single dark spot in the SW. In particular, no solution involving any bright spot could be found.

\subsection{Bayesian Spot Model Selection}

In general, the reduced $-\chi^{2}$ metric is ill-adapted to truly assess the relative probabilities of models (Marshall et al. 2006). The $\chi^{2}$ decrease that results from the addition of a new set of spot parameters can be due to modeling a real spot or simply to over-fitting, with emergence of artifacts due to imperfect $(u, v)$ coverage. Here, we present a general framework to treat the problem of fitting spots, based on Bayesian model selection. 
Model-fitting consists in estimating the most probable model coefficients $\boldsymbol{C}=\left\{c_{1}, c_{2}, \ldots c_{n}\right\}$ of a model $\boldsymbol{M}_{i}$. To do so, we actually maximize the posterior probability $p\left(\boldsymbol{C} \mid \boldsymbol{D}, \boldsymbol{M}_{i}\right)$ :

$$
p\left(\boldsymbol{C} \mid \boldsymbol{D}, \boldsymbol{M}_{i}\right)=\frac{p\left(\boldsymbol{D} \mid \boldsymbol{C}, \boldsymbol{M}_{i}\right) p\left(\boldsymbol{C} \mid \boldsymbol{M}_{i}\right)}{p\left(\boldsymbol{D} \mid \boldsymbol{M}_{i}\right)},
$$

where $p\left(\boldsymbol{D} \mid \boldsymbol{C}, \boldsymbol{M}_{i}\right)$ is the likelihood of the model coefficients, $p\left(\boldsymbol{C} \mid \boldsymbol{M}_{i}\right)$ is the prior probability of the coefficients, and $p\left(\boldsymbol{D} \mid \boldsymbol{M}_{\boldsymbol{i}}\right)$ is the marginal likelihood, also often called "Bayesian evidence" in astronomy. The evidence appears in Equation (1) as the denominator, its role being to normalize the posterior probability. For a given model $\boldsymbol{M}_{\boldsymbol{i}}$, the evidence is constant. To determine the $\boldsymbol{C}$ coefficients, it is then sufficient to maximize the denominator only: i.e., the likelihood (i.e., $\chi^{2}$ ) under prior constraints (mostly physical constraints such as positivity for the stellar diameters, spots within the stellar disks). However, when comparing two spot models, the ratio of their evidence is to be considered. The ratio of the probabilities of two models $\boldsymbol{M}_{1}$ and $\boldsymbol{M}_{2}$ given the data can be expressed as:

$$
\frac{p\left(\boldsymbol{M}_{1} \mid \boldsymbol{D}\right)}{p\left(\boldsymbol{M}_{2} \mid \boldsymbol{D}\right)}=\frac{p\left(\boldsymbol{M}_{1}\right)}{p\left(\boldsymbol{M}_{2}\right)} \frac{p\left(\boldsymbol{D} \mid \boldsymbol{M}_{\mathbf{1}}\right)}{p\left(\boldsymbol{D} \mid \boldsymbol{M}_{2}\right)},
$$

where $p\left(\boldsymbol{M}_{i}\right)$ is the a priori probability of model $\boldsymbol{M}_{i}$. As we have no specific preference for a model in the absence of data, $p\left(\boldsymbol{M}_{1}\right) / p\left(\boldsymbol{M}_{2}\right)=1$, and therefore, the most probable model corresponds to the model with the largest evidence. For a given data set, the evidence $Z\left(\boldsymbol{M}_{\boldsymbol{i}}\right)=p\left(\boldsymbol{D} \mid \boldsymbol{M}_{\boldsymbol{i}}\right)$ for model $\boldsymbol{M}_{\boldsymbol{i}}$ is defined as the marginalized likelihood:

$$
Z\left(\boldsymbol{M}_{\boldsymbol{i}}\right)=\int_{C_{1}} \ldots \int_{C_{n}} p(\boldsymbol{D} \mid \boldsymbol{C}, \boldsymbol{M}) p(\boldsymbol{C} \mid \boldsymbol{M}) d C_{1} \ldots d C_{n} .
$$

Computing the evidence and its associated error bar with good precision requires the exploration and integration of the posterior probability by specialized Monte Carlo Markov Chain (MCMC) algorithms. Our model-fitting code FITNESS was set to use the Importance Nested Sampling algorithm as implemented in the MultiNest library (Feroz \& Hobson 2008; Feroz et al. 2013) to compute the logarithm of the evidence $\log Z$ as well as its error bar. Because the ratio of evidence intervenes in Equation (2), differences of $\log Z$ encode the relative model probabilities and can be interpreted (with caution) using the Jeffrey's scale (Kass \& Raftery 1995). Contrary to the reduced$\chi^{2}, \log Z$ does not directly take into account the raw number of parameters, but it is based on their actual relevance to the fitting process. A good model has the minimal number of parameters required to explain the data (Occam's razor), which corresponds to a high $\log Z$. Bad models may be less predictive, or may be too generic due to the overabundance of parameters, and they are characterized by $\operatorname{low} \log Z$. We give the $\log Z$ for all our models in Tables 2 and 3 . For T Per, $\log Z$ points overwhelmingly in favor of the single bright spot model. Despite having better $\chi^{2}$, the single dark spot and the two-spot models are found to be much less probable. For RS Per, a comparison of the $\log Z$ for the spotted and non-spotted models indicates that the dark spot in the $\mathrm{SW}$ is probably real, though with a low confidence index. Both these results will be confirmed by image reconstruction in Section 4.

\subsection{Effective Surface Temperatures}

The effective temperature of a star $T_{\text {eff }}$ and its bolometric flux $F_{\text {bol }}$ follow the Stefan-Boltzmann law, $F_{\text {bol }}=\sigma T_{\text {eff }}^{4}$. The measured bolometric flux on Earth $f_{\text {bol }}$ is weaker by a factor of $\theta^{2} / 4$, where $\theta$ is the angular diameter of the star. The effective temperature is then given by

$$
T_{\mathrm{eff}}=\left(\frac{4 f_{\mathrm{bol}}}{\sigma_{B} \theta^{2}}\right)^{\frac{1}{4}}
$$

where $\sigma_{B}$ is the Stefan-Boltzmann constant. As recommended by Scholz \& Takeda (1987), $\theta$ is chosen to be the Rosseland angular diameter. Because our limb-darkening law is adjusted to fit directly to the Rosseland diameter (see Figure 4), it is equal to the diameter $\theta_{\star}$ of the best fits in Section 3.2.

To compute $f_{\text {bol }}$ for our targets, we derived the spectral energy distributions (SEDs) from public catalog records. Visible and near-ultraviolet data were obtained from Johnson UBVRI measurements (Johnson et al. 1966; Mendoza 1967; Morel \& Magnenat 1978; Ducati 2002) and from Slesnick et al. (2002). In addition, we used observations in the Geneva (Rufener 1999), Strömgren $u v b y \beta$ (Marco \& Bernabeu 2001), and Vilnius UPXYZVS systems (Straizys et al. 1995). The near-infrared data $(J H K s)$ was obtained from Morel \& Magnenat (1978) and Ducati (2002), as well as Two Micron Sky Survey (2MASS; Currie et al. 2010; Skrutskie et al. 2006). Because the 2MASS data is saturated for both stars, the corresponding $J H K$ fluxes are estimated using the less accurate profile method. Mid-infrared data was acquired by Infrared Space Observatory-SWS (Sloan et al. 2003), AKARI/IRC (Ishihara et al. 2010), WISE (Wright et al. 2010; see also http://wise2.ipac.caltech.edu/docs/release/ allsky/expsup/index.html), IRAS/LRS (Neugebauer et al. 1984), and the Midcourse Space Experiment Point Source Catalog (Egan et al. 2003). Far-infrared data came from IRAS (Neugebauer et al. 1984) and AKARI/FIS (Ishihara et al. 2010) observations.

The SEDs were de-reddened by adopting the extinction parameters found for the double cluster in Slesnick et al. (2002), i.e., a distance modulus of $11.85 \pm 0.05$ and color excesses of $E(B-V)=0.53 \pm 0.02$ for RS Per and $E(B-V)=$ $0.56 \pm 0.02$ for T Per. Based on the analysis of McCall (2004) and the recommendations of Massey et al. (2005), we attempted to de-redden the data using two different empirical laws for the reddening curves: first using the reddening curve from Cardelli et al. (1989) updated in the near-uv with coefficients from O'Donnell (1994), and with a total-to-selective ratio of absorption $R_{V}=4.15$; and second using the curve derived by Fitzpatrick (1999) with $R_{V}=3.8$. In both cases, we extended the de-reddening to the longest wavelengths, using equations from Chiar \& Tielens (2006) for $\lambda>5 \mu \mathrm{m}$. Figure 7 presents the final de-reddened SEDs using the Cardelli de-reddenning.

Both SEDs show significant amount of circumstellar emission in the mid-IR and far-IR, though is this much more pronounced for RS Per. The infrared excess of RS Per around $7.6 \mu \mathrm{m}$ is attributed to polycyclic aromatic hydrocarbon emission (Verhoelst et al. 2009), and a peak at $9.7 \mu \mathrm{m}$ indicates strong silicate emission (Speck et al. 2000). Both constitute evidence of ongoing dust production. Moreover, its far-infrared excess at $60 \mu \mathrm{m}$ is characteristic of extended circumstellar emission (Stencel et al. 1988, 1989) and indicates significant mass loss through a circumstellar outflow whose typical size can be estimated to about 4 arcmin (Stencel et al. 1989). In contrast, T Per does not appear as active but still displays broad dust emission between 9-13 $\mu \mathrm{m}$ range.

To determine the effective surface temperature, integration of the spectrophotometric data has to be restricted to photospheric emission. To this purpose, we fitted SATLAS models 

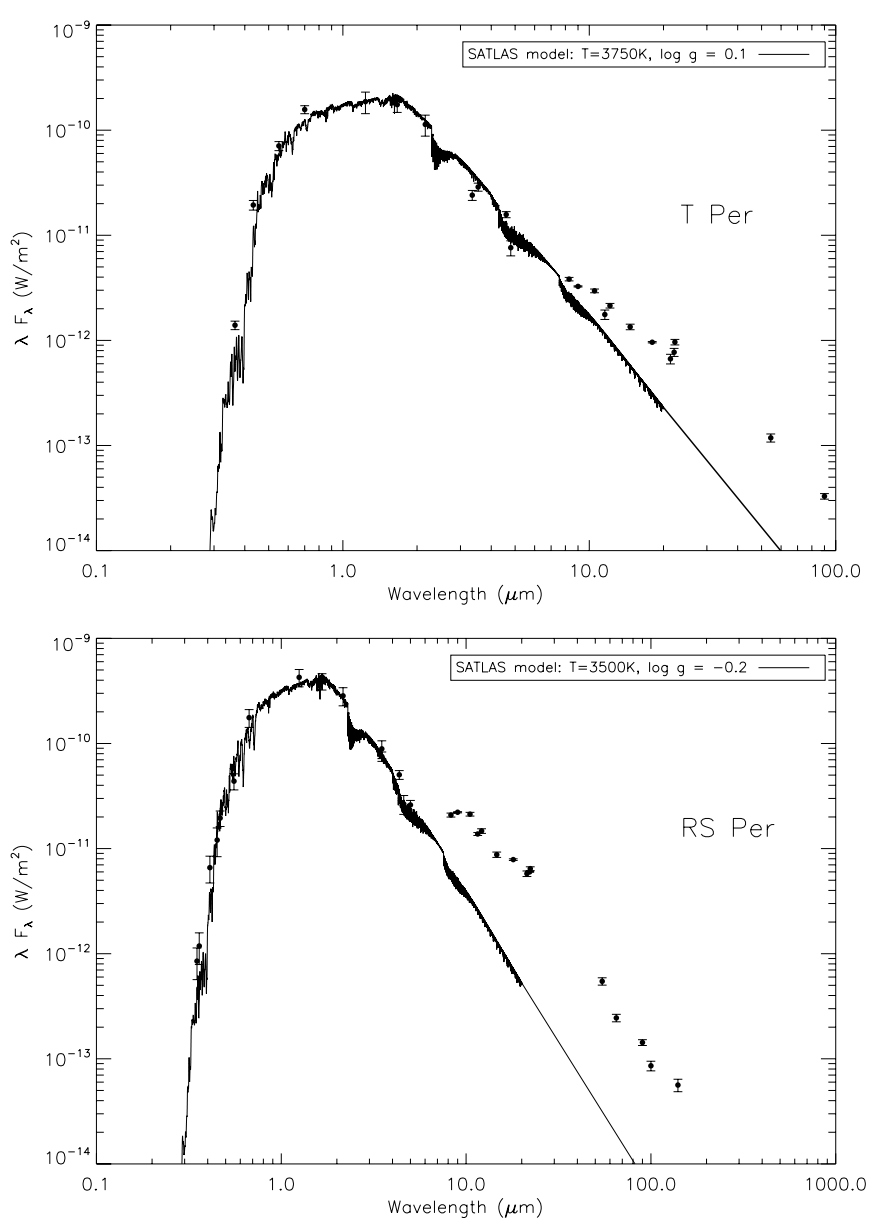

Figure 7. Spectral energy distributions of T Per (top) and RS Per (bottom). The plain lines show the dereddened SATLAS fit to the optical and near-IR parts of the spectra.

only to the ultraviolet, visible, and near-infrared wavelengths where the stellar photosphere clearly dominates the emission (i.e., $<3 \mu \mathrm{m}$ ). The SATLAS models were using ODF sampling with improved $\mathrm{H}_{2} \mathrm{O}$ lines and the following parameters: subsolar metallicity $[\mathrm{Fe} / \mathrm{Z}]=-0.5$, based on the assumed typical metallicity of the double cluster $[\mathrm{Fe} / \mathrm{Z}]=-0.35$ from Gonzalez \& Wallerstein (2000); a medium micro-turbulence level $\chi_{t}=3 \mathrm{~km} \mathrm{~s}^{-1}$ Gonzalez \& Wallerstein (2000); surface gravities $\log g=-0.5$ to 0.5 , and total mass $7-25 M_{\odot}$, typical of $\mathrm{M}$ supergiants; effective temperatures are in the range of $T=3300-4000 \mathrm{~K}$ based on previous estimates (Gonzalez \& Wallerstein 2000; Levesque et al. 2005; Verhoelst et al. 2009). In order to check the independence of our results from the specificities of SATLAS, we also fitted spherical MARCS models using the same parameters, and we obtained identical fit results. We found that the SED of T Per was fitted well by models with temperatures in the $3700 \mathrm{~K}-3800 \mathrm{~K}$ range and $\log g \simeq 0$; and for RS Per, temperature of $3500 \mathrm{~K}-3600 \mathrm{~K}$ and $\log g \simeq-0.5$.

To derive the bolometric fluxes, the photosphere SEDs were integrated with a Gaussian quadrature algorithm (in logarithm space). The SATLAS model was used instead of the actual SED only for wavelengths affected by circumstellar emission (i.e., $>3 \mu \mathrm{m}$ ). Our estimates of bolometric fluxes and the derived absolute bolometric magnitude) are presented in Table 4. We assumed an error floor of $1 \%$ uncertainty on bolometric fluxes to account for systematics in the absolute flux calibration of photometric data (see the discussion in Bessell

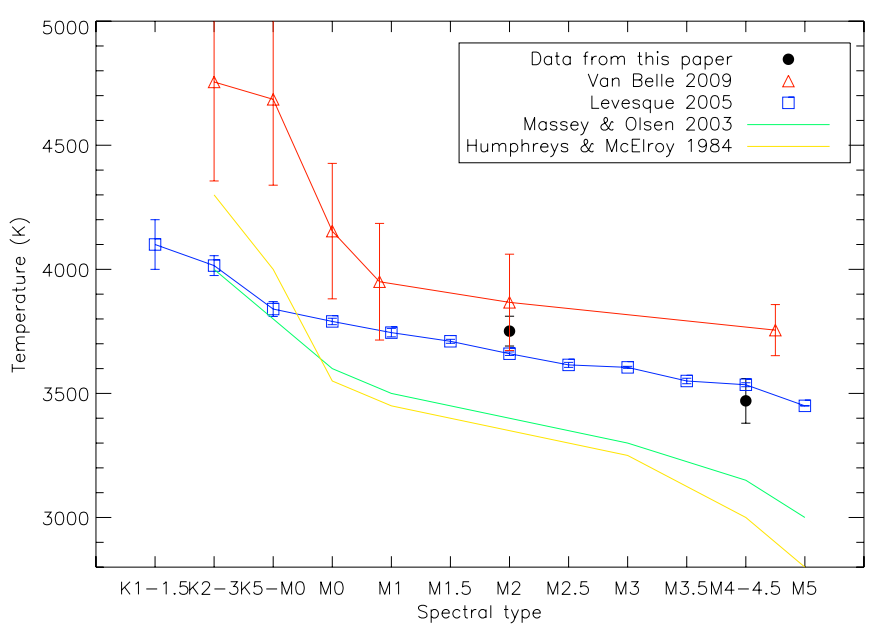

Figure 8. Effective temperatures for $\mathrm{T}$ Per and RS Per, compared to four temperature scales from the literature. Our results confirm the hotter scale of Levesque et al. (2005) and van Belle et al. (2009).

(A color version of this figure is available in the online journal.)

Table 4

Estimated Physical Parameters of T Per and RS Per

\begin{tabular}{lcc}
\hline \hline & T Per & RS Per \\
\hline$R_{\text {ross }}\left(R_{\odot}\right)$ & $510 \pm 20$ & $770 \pm 30$ \\
$M_{\text {bol }}$ & $-6.90 \pm 0.07$ & $-7.47 \pm 0.12$ \\
$T_{\text {eff }}(K)$ & $3750 \pm 60$ & $3470 \pm 90$ \\
$M_{\star}\left(M_{\odot}\right)$ & $9-12$ & $12-15$ \\
$\log L / L_{\odot}$ & $4.66 \pm 0.04$ & $4.89 \pm 0.05$ \\
$\log g(\mathrm{cgs})$ & $0.06 \pm 0.05$ & $-0.2 \pm 0.05$ \\
\hline
\end{tabular}

\& Murphy 2012). The probable inclusion of circumstellar emission into the integrated SED is also difficult to quantify without fully modeling the SED, but we estimated that it introduced an additional error of about $2 \%$. Our photometric error bars take into account the intrinsic variability of both targets as studied by Kiss et al. (2006), and the existence of contemporaneous visual, $B$ and $V$ band AAVSO data on RS Per. Overall, the errors are mostly dominated by the uncertainties arising from the de-reddening parameters: though relatively well-characterized, the extinction to the double cluster is considerable due to its distance, and the choice of the dereddening law significantly affects the bolometric magnitude. We note that our estimate of RS Per's absolute bolometric magnitude, $\mathrm{Mbol}=-7.47 \pm 0.12$, falls in the middle range of literature values: -7.21 in Slesnick et al. (2002), -7.48 in Gonzalez \& Wallerstein (2000), -7.74 in Verhoelst et al. (2009), and -8.15 in Levesque et al. (2005).

Using Equation (4), we derive the effective temperatures $T=3750 \pm 60 \mathrm{~K}$ for $\mathrm{T}$ Per and $T=3470 \pm 90 \mathrm{~K}$ for RS Per. Our error bars take into account both the spectrophotometric and interferometric errors, and as expected, they are mostly dependent on the latter. These temperatures are consistent with previous literature estimates (Gonzalez \& Wallerstein 2000; Slesnick et al. 2002; Verhoelst et al. 2009). Figure 8 presents our results in relation to four RSG temperature scales from the literature. Both scales from Humphreys \& McElroy (1984) and Massey \& Olsen (2003) were derived by averaging previous observations from public catalogs, but they most likely suffered from de-reddening issues, underestimating the RSG temperatures. Levesque et al. (2005) used spherical-geometry MARCS models (with the then-new opacity 
sampling method, later published in Gustafsson et al. 2008) and improved de-reddening of the sources, reconciling the observations with both predicted temperatures and evolutionary tracks. Finally, van Belle et al. (2009) estimated the temperature by fitting the SED with stellar templates derived from Pickles (1998) in place of synthetic models and independently estimated stellar diameters using the interferometer PTI. Note that the spatial resolution of our CHARA observations is at least twice that of PTI; therefore, it should provide more reliable diameter estimates.

Our results confirm the hotter temperature scales of RSGs, falling in-between the results of Levesque et al. (2005) and van Belle et al. (2009) for T Per, and slightly under the Levesque's curve for RS Per. Hence, and taking into account the limits of our analysis (noisy 2007 data compared to current CHARA/ MIRC data), we are reasonably confident in the quality of our temperature estimates.

\subsection{Linear Radii, Luminosity, Mass, and Surface Gravity}

Assuming a distance of $d=2345 \pm 55$ pc (Slesnick et al. 2002), our estimates of the linear radii are $R_{\text {ross }}=510 \pm 20 R_{\odot}$ for $\mathrm{T}$ Per and $R_{\text {ross }}=770 \pm 30 R_{\odot}$ for RS Per. This corresponds to luminosities $\log L / L_{\odot}=4.66 \pm 0.04$ for $\mathrm{T}$ Per and $\log L / L_{\odot}=4.90 \pm 0.05$ for RS Per, comparable to that of $\alpha$ Ori. To get estimates of the stellar masses, we used the most recent evolutionary tracks from Ekström et al. (2012) and the new Geneva tracks from Neugent et al. (2012), that both demonstrated their (relative) reliability on RSGs. The range of possible masses appears to be $M=9-12 M_{\odot}$ for $\mathrm{T}$ Per, and $M=12-15 M_{\odot}$ for RS Per, which translates into $\log g=0.06 \pm 0.05$ for $\mathrm{T}$ Per and $\log g=-0.2 \pm 0.05$ for RS Per. These results support the assumptions made during our selection of SATLAS models in Sections 3.1 and 3.4.

\section{IMAGE RECONSTRUCTION}

\subsection{Regularized Maximum Likelihood}

As four telescope data is secured on both objects, there exists enough phase information in our data sets to attempt "modelindependent image reconstruction." Here the prefix "modelindependent" signifies that the image reconstruction process will not rely on a specific astrophysical model. The target image $\boldsymbol{i}$ is modeled as an array of pixel fluxes $\widehat{\boldsymbol{i}}=\left\{i_{0}, \ldots i_{n-1}\right\}$.

As the data is assumed to be normally distributed, to each image we can associate a $\chi^{2}(\boldsymbol{i})$ metric that measure the distance between the observed data (power spectra and bispectra) and the same quantities derived from the current image. Maximizing the likelihood of the image by minimizing its $\chi^{2}$ unfortunately does not lead to reasonable images. The reason is that image reconstruction belongs to the class of "ill-posed" inverse problems: the number of pixels needed to reconstruct is typically a few thousand, while we only have a few hundred interferometric data points. Under these conditions, maximum likelihood leads to an overfitting the data. It is thus essential to "regularize" the solution by introducing reasonable but noncommittal prior expectations about the image. This is usually done through regularization functions that control the flux distribution within the image. In addition to preventing over-fitting, good regularizers fulfill other roles. As underlined during model-fitting, the $\chi^{2}$ is heavily multimodal. Most classic regularization function $R(i)$ effectively allow for discrimination between these local minima and thus eases minimization of Equation (5). In effect, regularizers help extrapolate the missing information from the phase lost to the atmosphere and the gaps in the data coverage of the $(u, v)$ plane. A competent choice of regularizers ensures that high frequencies are extrapolated well and that image reconstruction has demonstrated that it achieves super-resolution (Renard et al. 2011), i.e., the effective resolution of the reconstructed images is typically about three to four times greater than the interferometer resolution.

This regularized maximum likelihood approach constitutes the current framework for image reconstruction in optical interferometry (Baron et al. 2010; Thiébaut \& Giovannelli 2010). Formally, the target image minimizes the sum of the $\chi^{2}(i)$ metric and of $K$ regularizers $R_{k}(i)$ :

$$
\widehat{\boldsymbol{i}}=\underset{i \in \mathbb{R}^{n}}{\operatorname{argmin}}\left\{\chi^{2}(\boldsymbol{i})+\sum_{k=1}^{K} \mu_{k} R_{k}(\boldsymbol{i})\right\},
$$

under the constraints of image positivity $\left(\forall n, i_{n} \geqslant 0\right)$ and of normalization of the image to unity $\left(\sum_{n} i_{n}=1\right)$. The factors $\mu_{k}$ in Equation (5) control the relative weight of the $\chi^{2}$ and regularization terms.

\subsection{Reconstructing Spotted Stars with Current Software}

Reconstructing spotted stars is currently difficult with available software. To date, the only published model-independent interferometric reconstructions of stellar spots are that of the large convection cells of $\alpha$ Ori (Young et al. 2000; Haubois et al. 2009; Chiavassa et al. 2010b) and of VX Sgr (Chiavassa et al. 2010a). Resolving spots entails that the stellar disk is proportionally much larger, which implies very low visibility amplitudes, and consequently bad signal-to-noise. Moreover, the conventional convex approximations of the $\chi^{2}$ expression should then be ruled out. And as exemplified by the difficult reconstruction of VX Sgr, the minimization of the non-convex $\chi^{2}$ is very prone to appearance of artifacts when using conventional tools such as BSMEM (Baron et al. 2010) or MIRA (Thiébaut \& Giovannelli 2010).

To solve this issue, we suggest the use of non-convex reconstruction codes, such as those based on an MCMC approach. In this paper, the software SQUEEZE (Baron et al. 2010) was used to obtain the reconstructions presented in this paper. SQUEEZE uses parallel tempering to tentatively find the global minimum of the criterion in Equation (5) and, therefore, is well-adapted to non-convex problems. SQUEEZE is multi-threaded, with each thread conducting minimization by simulated annealing at a different temperature and starting with a different random seed. Compared to its predecessor MACIM (Ireland et al. 2006), it is less sensitive to the initial condition of the Markov Chains (i.e., the starting image). Thus, the quality of its reconstructions mostly depends on the choice of regularization.

To select the best regularizer, we generated a synthetic test data set simulating the observation of a spotted star using the OIFITS-SIM tools (https://github.com/bkloppenborg/ oifits-sim). The original image used to create the data was chosen as the T Per "bright spot" model from Figure 5, and we use the same $(u, v)$ coverage and signal-to-noise as the actual $\mathrm{T}$ Per data. We then reconstructed the stellar surface using the two most successful regularizers as benchmarked by Renard et al. (2011): maximum entropy and total variation (Rudin et al. 1992). Maximum entropy was implemented using the multiplicity expression from Sutton \& Wandelt (2006), which is well adapted to our MCMC implementation:

$$
R_{\Gamma}(\boldsymbol{i})=\sum_{n} \log \Gamma\left(i_{n}+1\right)
$$


where $i_{n}$ is the flux in pixel $n$. Total variation (hereafter, TV) is defined as the $\ell_{1}$ norm of the spatial gradient $g$ :

$$
R_{\mathrm{TV}}(\boldsymbol{i})=\ell_{1}(\boldsymbol{g})=\sum_{n}\left|g_{n}\right|
$$

Several practical expressions are available to discretize $\boldsymbol{g}$ on the image grid. In the context of this paper, we implemented the classic isotropic formulation of $g$, i.e., for each pixel coordinate $(n, m)$ in the two-dimensional image $\boldsymbol{i}$, the local gradient was given by

$$
g_{n, m}(\boldsymbol{i})=\sqrt{\left|i_{n+1, m}-i_{n, m}\right|^{2}+\left|i_{n, m+1}-i_{n, m}\right|^{2}} .
$$

Figure 9 compares regularization obtained with both these regularizers on our synthetic data set (the full reconstruction procedure is detailed in Section 4.4). Our results demonstrate that the maximum entropy image suffers from several flaws: the stellar background is excessively non-uniform, and the precise location of the spot is lost. The total variation reconstruction is definitively superior on both aspects. Moreover, and unlike maximum entropy, total variation does not require an additional prior to constrain the flux to stay within a given diameter. The good performance of total variation is in line with the empirical results of Renard et al. (2011) but also with the theoretical predictions. Total variation is indeed a direct application of the Compressed Sensing theory, a recent mathematical framework that supersedes the conventional Shannon sampling theorem when applied to sparse images, i.e., images that may be described with small number of non-zero coefficients in some give basis. Here, on first order, our model spotted star consists of a (mostly) uniform disk with compact spots or cells. The spatial gradient of the image is sparse, with only the perimeters of the stellar disk and the spots as non-zero components. Total variation enforces the sparsity of the spatial gradient so that the reconstruction is a piecewise constant with sharp transitions, though this is not apparent on Figure 9 as these images are actually Markov Chain averages as explained further in Section 4.4.

\subsection{A Novel Regularizer for Spotted Stars}

To derive a novel regularizer for use on spotted stars, we built upon the idea of spatial gradient sparsity, adding two noncommittal requirements. The first requirement is that for a given flux to distribute into possible spots, the regularizer should prefer a single spot to two spots, as per Occam's razor prescription. The second requirement is that for a given flux to attribute to a spot, the size of the spot should be determined solely by the data. This implies that the regularizer value should be independent of the spot size.

It is straightforward to demonstrate that TV violates this second requirement. Let us consider an idealized stellar disk and two cases: either two small spots of diameter $D$, or a single large spot of diameter two dimensions. Let us assume the brightness distribution of the disk and the spots as uniform, so that the spatial gradient is null everywhere except on the perimeters of these components. The actual contribution of the spots to the spatial gradient is then proportional to the spot perimeters - equal to $2 \pi D$ in both cases - multiplied by the flux density for each case. Assuming that a combined flux $F$ is emitted by the spots, the spot flux density is then $F /\left(\pi D^{2}\right)$ for the single spot case, and $2 F /\left(\pi D^{2}\right)$ for the two spot case. Consequently, the total variation is $T V$ (one spot) $=F / D$ for a single spot and $T V(2$ spots $)=2 F / D$ for two spots. While this implies that TV does favor a single spot, this also demonstrates that the regularization depends on the size of the spot, and therefore, it may bias a reconstruction toward larger spots.

In contrast the regularizer $R_{\text {spot }}$ defined by

$$
R_{\text {spot }}(\boldsymbol{i})=\ell_{\frac{1}{2}}(\boldsymbol{g})=\left(\sum_{n} \sqrt{\left|g_{n}\right|}\right)^{2}
$$

meets both our requirements for an ideal regularizer, with $R_{\text {spot }}($ one spot $)=4 \pi F$ and $R_{\text {spot }}($ two spots $)=8 \pi F$. Figure 9 confirms our analysis, and our spot regularizer demonstrates a significant improvement over total variation.

\subsection{Reconstruction Procedure and Results}

The instrumental resolution is given by the largest CHARA baseline in our data sets (S2-W1 or E2-W1, 2250 meters), corresponding to 1.3 mas in $H$ band. Taking into account a super-resolution factor of four, the effective resolution of the reconstructed images should be about 0.3 mas. In order to avoid excessive pixellation of the images, the actual resolution of the reconstruction was set to 0.1 mas. We ran five batches of multithreaded SQUEEZE with 16 threads each, corresponding to a total of 80 independent Markov chains that were averaged to reconstruct the final images. The number of pixel elements in each chain was set to 5000, with a length of 500 iterations. In addition to the spot regularizer derived in the previous section, we made use of the fitting results from Section 3.1 to constrain the reconstruction. The fitted limb-darkening disks were used to initialize the chains to sensible starting points. The factors $\mu_{k}$ were chosen so that the actual reduced $\chi^{2}$ is roughly unity for the reconstructed image. The final reconstructions are presented on Figure 10. For T Per, the spot location in the northwest quadrant agrees with the conclusions of Bayesian model selection. For RS Per, there is indeed a darker area in the southwest. However, the correct interpretation is unclear: this may be a dark spot, or most of the surface could be understood as a temporary hot convection cell. Without data outside the 2007 July/August period, we cannot conclude from this single RS Per image. To exclude the possibility that the surface features on Figure 10 are due to $(u, v)$ coverage or to noisy data, we ran an "artifact test" on both targets. We generated synthetic observations of the limbdarkening disks derived from model fitting, with exactly the same $(u, v)$ coverage and signal-to-noise as the real data sets. We then reconstructed the images using the same procedure outlined above, and we found that the reconstructions did not display any significant surface features.

\section{DISCUSSION AND CONCLUSION}

We have analyzed the CHARA/MIRC data taken in $H$ band on two RSGs T Per and RS Per and presented evidence for the existence of spots on their surfaces. For this, we developed a set of tools dedicated to the analysis of spotted stars. As the utility of simple model-fitting procedures is limited for spotted stars, we demonstrated that Bayesian model selection is capable of assessing the relative probabilities of various models, the Bayesian evidence constituting a more reliable metric than the reduced $\chi^{2}$. Our results on T Per confirm that hotspots can indeed be observed in $H$ band. If considered together with similar results on $\alpha$ Ori by (Haubois et al. 2009) and on VX Sgr by (Chiavassa et al. 2010b), it seems we should expect a significant proportion of RSGs to have bright spots, as is thought 

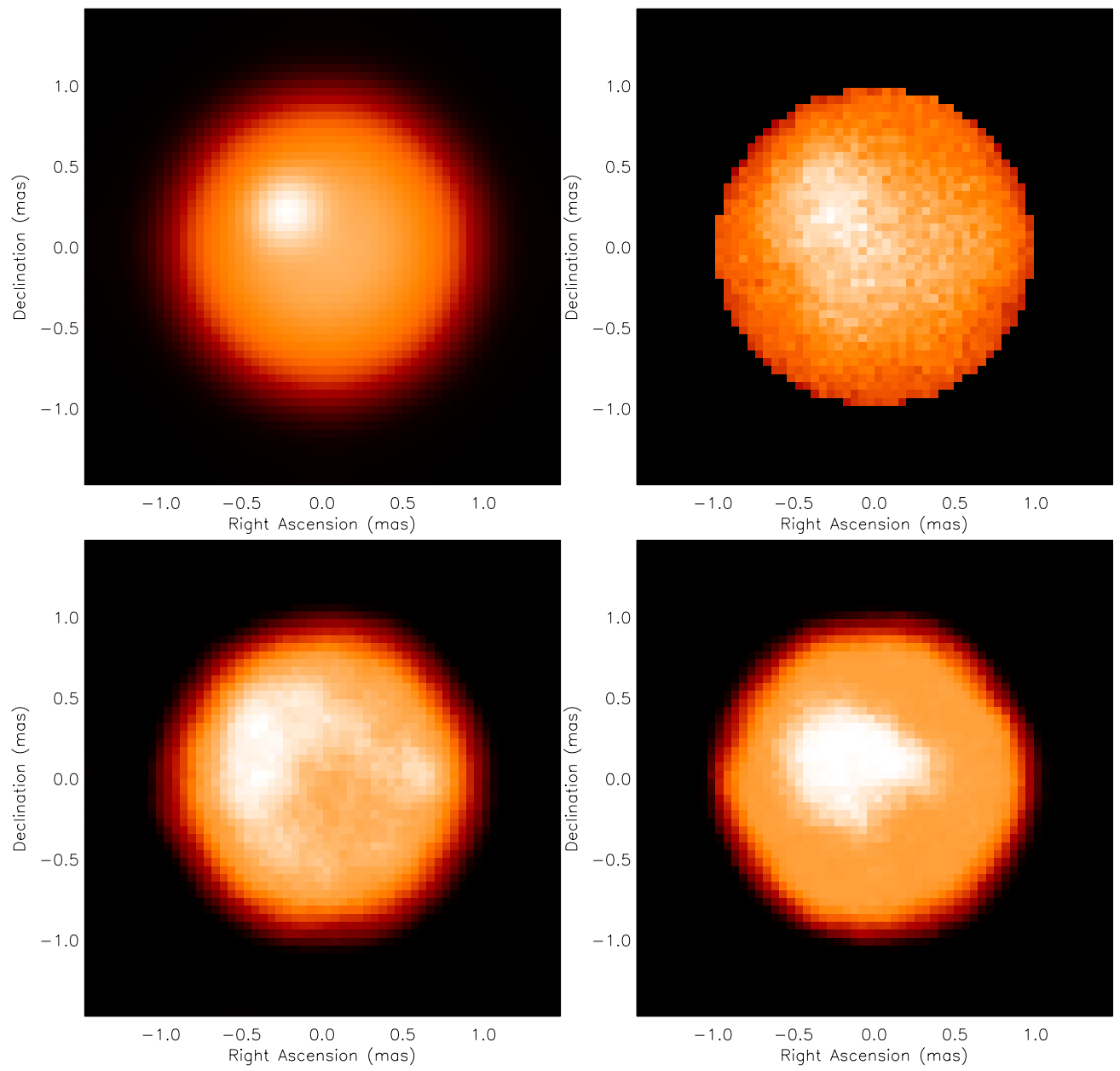

Figure 9. Reconstructions of a synthetic spotted star with the same $u v$ coverage and signal-to-noise as the T Per data. Top left: the original image convolved to the expected effective resolution (using a super-resolution factor of three); top right: reconstruction regularized by maximum entropy and a prior constraining the flux to stay within the stellar diameter; bottom left: reconstruction regularized by total variation; bottom right: reconstruction regularized by the spot regularizer presented in Section 4.3.

(A color version of this figure is available in the online journal.)
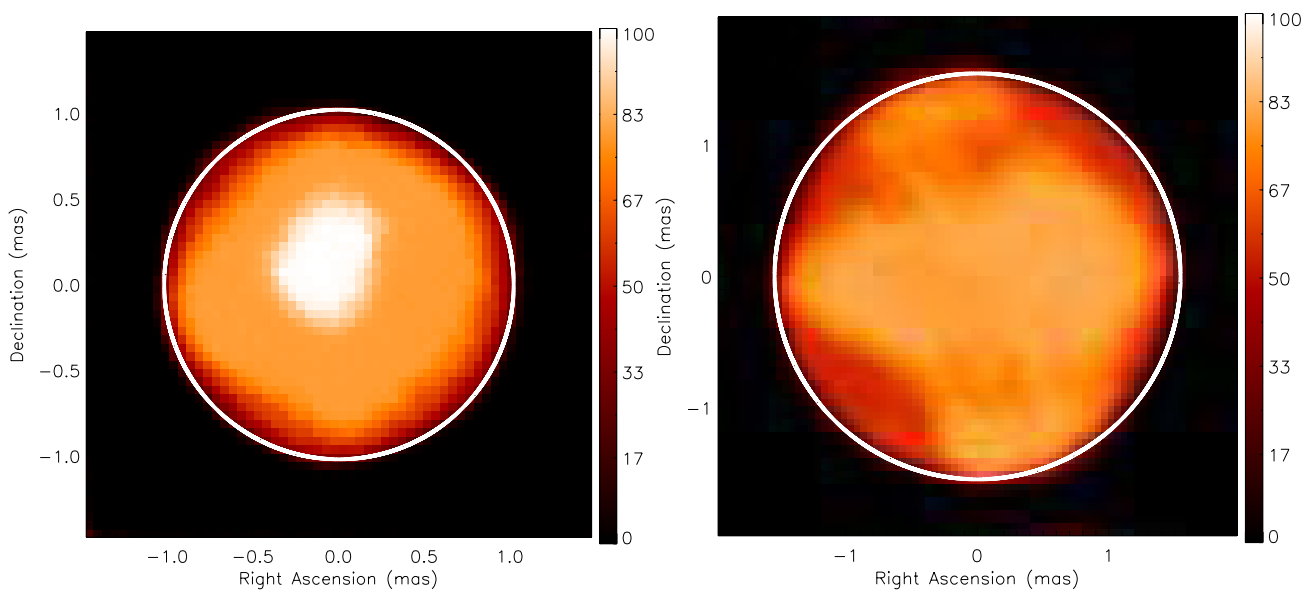

Figure 10. Reconstructed images of T Per (left) and RS Per (right) with the SQUEEZE-MCMC engine and the "spot regularizer" presented in Section 4.3. The angular diameters estimated by model-fitting are indicated by white circles.

(A color version of this figure is available in the online journal.)

to be the case for AGB stars (Ragland et al. 2006). Considering the typical continuum opacity curves in such cool atmospheres (Woodruff et al. 2009), the continuum opacity should be close to the minimum in the $H$ band, where our MIRC observations took place. These spots must be generated very close to the photosphere, and therefore, it seems currently doubtful that their enhanced contrast may be explained by opacity effects. As the correct approach to model these objects is not really to model spots but to interpret the surface in terms of convective cells using 3D models (Chiavassa et al. 2010a), inhomogeneous 
granulation temperatures may explain the spots. Our detection of a "dark spot" on RS Per probably corresponds to a cooler granulation, imaged with the reduced dynamic contrast typical of current interferometry.

We also found that classic regularizers are hardly adequate to reconstruct model-independent images of spotted surfaces. Hence, we derived a novel regularizer tailored for this task, based on simple Compressed Sensing and Occam's razor principles. Our reconstructions of $\mathrm{T}$ Per and RS Per were found to essentially agree with the Bayesian spot selection.

It should be underlined that the data quality from MIRC circa 2007 was a major limiting factor in the present analysis. Fortunately since 2007, the MIRC combiner underwent a series of hardware upgrades which drastically improved its performance. MIRC-6T can now simultaneously combine all six CHARA telescopes with thrice higher signal-to-noise and much lower systematic errors. A survey of several RSGs over longer periods of time with MIRC-6T would allow us to conclude on whether spots on M supergiants are ubiquitous, and in particular, if a relationship can be found between circumstellar activity/ infrared excess and the complexity of observed surface features. Our future work will thus focus on the analysis of new RSG data collected with MIRC-6T, with a systematic application of Bayesian model selection to 3D hydrodynamical models, and the development of better reconstruction algorithms for RSGs.

We would like to thank the anonymous reviewer for his/her very constructive comments.

The authors would like to acknowledge funding from the NSF through award AST-0807577 to the University of Michigan, as well as funding by the Australian Research Council and the "Lendület Fiatal Kutatói Program" of the Hungarian Academy of Sciences for the initial work on these objects.

This work is based on observations collected at the Center for High Angular Resolution Astronomy (CHARA) operated by the Georgia State University at Mt. Wilson, California. This research also relied on observations with $A K A R I$, a JAXA project with the participation of ESA, and made use of data products from the Wide-field Infrared Survey Explorer (a joint project of the University of California, Los Angeles, and the Jet Propulsion Laboratory/California Institute of Technology, funded by the National Aeronautics and Space Administration), and from the Two Micron All Sky Survey (a joint project of the University of Massachusetts and the Infrared Processing and Analysis Center/California Institute of Technology, funded by the National Aeronautics and Space Administration and the National Science Foundation).

\section{REFERENCES}

Airapetian, V., Carpenter, K. G., \& Ofman, L. 2010, ApJ, 723, 1210

Aurière, M., Donati, J.-F., Konstantinova-Antova, R., et al. 2010, A\&A, 516, L2

Barnes, T. G., Evans, D. S., \& Moffett, T. J. 1978, MNRAS, 183, 285

Baron, F., Monnier, J., \& Kloppenborg, B. 2010, Proc. SPIE, 7734, 77342I

Bessell, M., \& Murphy, S. 2012, PASP, 124, 140

Buscher, D. F., Baldwin, J. E., Warner, P. J., \& Haniff, C. A. 1990, MNRAS $245,7 \mathrm{P}$

Cardelli, J. A., Clayton, G. C., \& Mathis, J. S. 1989, ApJ, 345, 245

Castelli, F., \& Kurucz, R. L. 2003, in IAU Symp. 210, Modelling of Stellar Atmospheres, ed. N. Piskunov, W. W. Weiss, \& D. F. Gray (Cambridge: Cambridge Univ. Press), 20

Chiar, J. E., \& Tielens, A. G. G. M. 2006, ApJ, 637, 774

Chiavassa, A., Freytag, B., Masseron, T., \& Plez, B. 2011, A\&A, 535, A22

Chiavassa, A., Haubois, X., Young, J. S., et al. 2010a, A\&A, 515, A12

Chiavassa, A., Lacour, S., Millour, F., et al. 2010b, A\&A, 511, A51
Chiavassa, A., Plez, B., Josselin, E., \& Freytag, B. 2009, A\&A, 506, 1351

Cuntz, M. 1997, A\&A, 325, 709

Currie, T., Hernandez, J., Irwin, J., et al. 2010, ApJS, 186, 191

Danchi, W. C., Bester, M., Degiacomi, C. G., Greenhill, L. J., \& Townes, C. H. 1994, AJ, 107, 1469

Ducati, J. R. 2002, yCat, 2237, 0

Egan, M. P., Price, S. D., Kraemer, K. E., et al. 2003, yCat, 5114, 0

Ekström, S., Georgy, C., Eggenberger, P., et al. 2012, A\&A, 537, A146

Feroz, F., \& Hobson, M. P. 2008, MNRAS, 384, 449

Feroz, F., Hobson, M. P., Cameron, E., \& Pettitt, A. N. 2013, arXiv:1306.2144 Fitzpatrick, E. L. 1999, PASP, 111, 63

Freytag, B., Steffen, M., \& Dorch, B. 2002, AN, 323, 213

Gonzalez, G., \& Wallerstein, G. 2000, AJ, 119, 1839

Gray, D. F. 2008, AJ, 135, 1450

Grunhut, J. H., Wade, G. A., Hanes, D. A., \& Alecian, E. 2010, MNRAS, 408, 2290

Gustafsson, B., Bell, R. A., Eriksson, K., \& Nordlund, A. 1975, A\&A, 42, 407

Gustafsson, B., Edvardsson, B., Eriksson, K., et al. 2008, A\&A, 486, 951

Haniff, C. A. 1991, JOSAA, 8, 134

Haubois, X., Perrin, G., Lacour, S., et al. 2009, A\&A, 508, 923

Hauschildt, P. H., \& Baron, E. 1999, JCoAM, 109, 41

Hestroffer, D. 1997, A\&A, 327, 199

Humphreys, R., \& McElroy, D. 1984, ApJ, 284, 565

Ireland, M. J., Monnier, J. D., \& Thureau, N. 2006, Proc. SPIE, 6268, 62681T

Ishihara, D., Onaka, T., Kataza, H., et al. 2010, yCat, 2297, 0

Johnson, H. L., Mitchell, R. I., Iriarte, B., \& Wisniewski, W. Z. 1966, CoLPL, 4,99

Josselin, E., \& Plez, B. 2007, A\&A, 469, 671

Kass, R. E., \& Raftery, A. E. 1995, JASA, 90, 773

Kervella, P., \& Fouqué, P. 2008, A\&A, 491, 855

Kervella, P., Perrin, G., Chiavassa, A., et al. 2011, A\&A, 531, A117

Kiss, L. L., Szabó, G. M., \& Bedding, T. R. 2006, MNRAS, 372, 1721

Kurucz, R. L. 1992, in IAU Symp. 149, The Stellar Populations of Galaxies, ed. B. Barbuy \& A. Renzini (Cambridge: Cambridge Univ. Press), 225

Lee, H.-T., \& Lim, J. 2008, ApJ, 679, 1352

Lester, J. B., \& Neilson, H. R. 2008, A\&A, 491, 633

Levesque, E. M. 2010, NewAR, 54, 1

Levesque, E. M., Massey, P., Olsen, K. A. G., et al. 2005, ApJ, 628, 973

Levesque, E. M., Massey, P., Olsen, K. A. G., et al. 2006, ApJ, 645, 1102

Marco, A., \& Bernabeu, G. 2001, A\&A, 372, 477

Marshall, P., Rajguru, N., \& Slosar, A. 2006, PhRvD, 73, 067302

Massey, P., Levesque, E. M., Plez, B., \& Olsen, K. A. G. 2008, in IAU Symp. 250, Massive Stars as Cosmic Engines, ed. F. Bresolin, P. A. Crowther, \& J. Puls (Cambridge: Cambridge Univ. Press), 97

Massey, P., \& Olsen, K. A. G. 2003, AJ, 126, 2867

Massey, P., Plez, B., Levesque, E. M., et al. 2005, ApJ, 634, 1286

McCall, M. L. 2004, AJ, 128, 2144

Meimon, S., Mugnier, L. M., \& Le Besnerais, G. 2005, JOSAA, 22, 2348

Meimon, S., Mugnier, L. M., \& Le Besnerais, G. 2008, JOSAA, 26, 108

Mendoza, E. E. 1967, BOTT, 4, 149

Mérand, A., Bordé, P., \& Coudé du Foresto, V. 2005, A\&A, 433, 1155

Meynet, G., \& Maeder, A. 2003, A\&A, 404, 975

Monnier, J., Schöller, M., \& Danchi, W. C. 2006, Proc. SPIE, 6268, 62681P

Monnier, J. D., Berger, J.-P., Millan-Gabet, R., \& ten Brummelaar, T. A. 2004a, Proc. SPIE, 5491, 1370

Monnier, J. D., Millan-Gabet, R., Tuthill, P. G., et al. 2004b, ApJ, 605, 436

Monnier, J. D., Zhao, M., Pedretti, E., et al. 2007, Sci, 317, 342

Monnier, J. D., Zhao, M., Pedretti, E., et al. 2008, Proc. SPIE, 7013, 701302

Morel, M., \& Magnenat, P. 1978, A\&AS, 34, 477

Neilson, H. R., \& Lester, J. B. 2013, A\&A, 554, A98

Neugebauer, G., Habing, H. J., van Duinen, R., et al. 1984, ApJL, 278, L1

Neugent, K. F., Massey, P., Skiff, B., \& Meynet, G. 2012, ApJ, 749, 177

O’Donnell, J. E. 1994, ApJ, 422, 158

Ohnaka, K., Hofmann, K.-H., Benisty, M., et al. 2009, A\&A, 503, 183

Ohnaka, K., Hofmann, K.-H., Schertl, D., et al. 2013, A\&A, 555, A24

Ohnaka, K., Weigelt, G., Millour, F., et al. 2011, A\&A, 529, A163

Percy, J. R., \& Sato, H. 2009, JRASC, 103, 11

Perrin, G. 2003, A\&A, 400, 1173

Pickles, A. J. 1998, PASP, 110, 863

Plez, B. 2003, in ASP Conf. Ser. 298, GAIA Spectroscopy: Science and

Technology, ed. U. Munari (San Francisco, CA: ASP), 189

Ragland, S., Traub, W. A., Berger, J.-P., et al. 2006, ApJ, 652, 650

Renard, S., Thiébaut, E., \& Malbet, F. 2011, A\&A, 533, A64

Rudin, L., Osher, S., \& Fatemi, E. 1992, PhyD, 60, 259

Rufener, F. 1999, yCat, 2169, 0

Sargent, B. A., Srinivasan, S., \& Meixner, M. 2011, ApJ, 728, 93

Scholz, M., \& Takeda, Y. 1987, A\&A, 186, 200 
Schwarzschild, M. 1975, ApJ, 195, 137

Skrutskie, M. F., Cutri, R. M., Stiening, R., et al. 2006, AJ, 131, 1163

Slesnick, C., Hillenbrand, L., \& Massey, P. 2002, ApJ, 576, 880

Sloan, G. C., Kraemer, K. E., Price, S. D., \& Shipman, R. F. 2003, ApJS, 147,379

Speck, A. K., Barlow, M. J., Sylvester, R. J., \& Hofmeister, A. M. 2000, A\&AS, 146,437

Stencel, R. E., Pesce, J. E., \& Bauer, W. H. 1989, AJ, 97, 1120

Stencel, R. E., Pesce, J. E., \& Hagen Bauer, W. 1988, AJ, 95, 141

Stothers, R. B. 2010, ApJ, 725, 1170

Straizys, V., Kazlauskas, A., Jodinskiene, E., \& Bartkevicius, A. 1995, yCat, 2157, 0

Sutton, E. C., \& Wandelt, B. D. 2006, ApJS, 162, 401

ten Brummelaar, T. A., McAlister, H. A., Ridgway, S. T., et al. 2005, ApJ, 628,453
Thiebaut, E. 2008, Proc. SPIE, 7013, 701311

Thiébaut, E., \& Giovannelli, J. 2010, ISPM, 27, 97

Tuthill, P., Haniff, C., \& Baldwin, J. 1997, MNRAS, 285, 529

van Belle, G. T., Creech-Eakman, M. J., \& Hart, A. 2009, MNRAS, 394,1925

Verhoelst, T., van der Zypen, N., Hony, S., et al. 2009, A\&A, 498, 127

Wittkowski, M., Aufdenberg, J. P., Driebe, T., et al. 2006, A\&A, 460, 855

Wittkowski, M., Aufdenberg, J. P., \& Kervella, P. 2004, A\&A, 413, 711

Wittkowski, M., Hauschildt, P. H., Arroyo-Torres, B., \& Marcaide, J. M. 2012, A\&A, 540, L12

Woodruff, H. C., Ireland, M. J., Tuthill, P. G., et al. 2009, ApJ, 691, 1328

Wright, E. L., Eisenhardt, P. R. M., Mainzer, A. K., et al. 2010, AJ, 140, 1868

Yang, M., \& Jiang, B. W. 2012, ApJ, 754, 35

Young, J. S., Baldwin, J. E., Boysen, R. C., et al. 2000, MNRAS, 315, 635

Zhao, M., Monnier, J. D., Che, X., et al. 2011, PASP, 123, 964 